Trauma Berufskrankh 2007 · 9[Suppl 3]:

S346-S348

DOI 10.1007/s10039-007-1302-8

Online publiziert: 26. Oktober 2007

(c) Springer Medizin Verlag 2007
H.-J. Wirthl

Landesverband Hessen-Mittelrhein und Thüringen

der gewerblichen Berufsgenossenschaften, Mainz

\title{
Die persönliche Leistungserbringung in Klinik und Praxis
}

\section{Auftrag und Ziel der gesetzlichen Unfallversicherung}

Die Träger der gesetzlichen Unfallversicherung (UV-Träger) haben die wichtige Aufgabe, nach Arbeitsunfällen die Gesundheit und die Leistungsfähigkeit der Versicherten mit allen geeigneten Mitteln wieder herzustellen [ $\$ 1$ Nr. 2 Sozialgesetzbuch (SGB) VII]. Die optimale medizinische Betreuung des Versicherten sowie seine berufliche und soziale Wiedereingliederung stehen stets im Vordergrund aller Bemühungen. Ziel ist die bestmögliche Wiedereingliederung in Arbeit, Beruf und Gesellschaft. Dazu haben die UVTräger den gesetzlichen Auftrag, alle Maßnahmen zu treffen, durch die eine möglichst frühzeitig nach dem Versicherungsfall einsetzende und sachgemäße Heilbehandlung und, soweit erforderlich, besondere unfallmedizinische Behandlung gewährleistet wird (\$ 34 SGB VII). Die Sicherstellung einer frühzeitig einsetzenden unfallchirurgischen Versorgung auf qualitativ hohem Niveau durch besonders ausgewählte Ärzte und Krankenhäuser ist dabei Garant für die Erfüllung des gesetzlichen Auftrags zur „Heilbehandlung mit allen geeigneten Mitteln“. Die UV-Träger sind ermächtigt, die von den Ärzten und Krankenhäusern zu erfüllenden Voraussetzungen im Hinblick auf die fachliche Befähigung, die sächliche und personelle Ausstattung sowie die zu übernehmenden Pflichten festzulegen. Von diesem Organisationsrecht haben die UV-Träger Gebrauch gemacht und ein umfangreiches und bewährtes Instrumentarium geschaffen. Die im Bereich der Akutversorgung nach einem Arbeitsunfall bedeutendsten Verfahren sind

- das Durchgangsarztverfahren,

- das Verletzungsartenverfahren sowie

- das H-Arzt-Verfahren.

\section{Grundlagen der Beteiligung von Ärzten an der Heilbehandlung}

Die Landesverbände der gewerblichen Berufsgenossenschaften beteiligen Ärzte an der bg-lichen Heilbehandlung, wenn sie die in den "Anforderungen der gesetzlichen Unfallversicherungsträger nach $₫ 34$ SGB VII „festgelegten Voraussetzungen erfüllen. Hiernach wird nur der Arzt beteiligt, der gewährleistet, dass Qualität und Wirksamkeit der Leistungen zur Heilbehandlung und Rehabilitation dem allgemein anerkannten Stand der medizinischen Erkenntnisse entsprechen und den medizinischen Fortschritt berücksichtigen. Dazu gehören eine bestimmte sächliche und personelle Ausstattung der Praxis, eine besondere unfallmedizinische Qualifikation der Ärzte auf Facharztniveau und die Erfüllung besonderer Pflichten. Durchgangs (D)- und H-Ärzte sind verpflichtet, ihre Tätigkeit persönlich und unter Anwendung des Ärztevertrags auszuüben.

Basis jedes Beteiligungsvertrags ist demnach außer den "Anforderungen“ der UV-Träger auch der „Vertrag gemäß $\$ 34$ Abs. 3 SGB VII über die Durchführung der Heilbehandlung, die Vergütung der Ärzte sowie die Art und Weise der Abrechnung der ärztlichen Leistungen (Vertrag Ärzte/Unfallversicherungsträger)“. Auch dieser Ärztevertrag verpflichtet in $\$ 24$ Abs. 3 D- und H-Ärzte dazu, die Tätigkeit persönlich auszuüben. Dies gilt auch für die Auswertung der Befunde beim Einsatz der Röntgendiagnostik und anderer bildgebender Verfahren.

\section{Unfallärztliche Bereitschaft des Durchgangsarztes}

Dem D-Arzt kommt bei der Behandlung von Arbeitsunfallverletzten eine besondere Stellung im Zusammenhang mit der Steuerung des Heilverfahrens zu. Insbesondere bei über den Unfalltag hinaus bestehender Arbeitsunfähigkeit oder einer voraussichtlich länger als eine Woche andauernden Behandlungsbedürftigkeit haben Arbeitgeber und Hausärzte die Verpflichtung, dafür zu sorgen, dass sich Arbeitsunfallverletzte unverzüglich einem D-Arzt vorstellen. Dieser entscheidet, ob allgemeine (hausärztliche) Heilbehandlung ausreicht oder besondere Heilbehandlung erforderlich ist. Besondere Heilbehandlung kann der D-Arzt in weitgehend freiem Ermessen einleiten und durchführen, wenn Art und Schwere der Verletzung es erfordern. Aber auch in Fällen der allgemeinen Behandlung ist es seine Aufgabe, den Heilverlauf im Rahmen eines Nachschauverfahrens zu überwachen.

Um diese auch gegenüber den $\mathrm{H}$ Ärzten herausgehobene Stellung im bg-li- 
chen Heilverfahren wahrnehmen zu können, ist die persönliche Leistungserbringung selbstverständliche Voraussetzung. Dies wiederum erfordert natürlich die persönliche Anwesenheit des D-Arztes in seiner Praxis, zumindest während der allgemein üblichen Arbeitszeiten. Die UVTräger haben deshalb eine sog. Präsenzbereitschaft des D-Arztes montags bis freitags von 8.00 bis 18.00 Uhr und an Samstagen von 8.00 bis 13.00 Uhr festgelegt. An Samstagen besteht jedoch die Möglichkeit, Vertretungsregelungen mit anderen D-Ärzten am Ort zu vereinbaren.

Die Präsenzbereitschaft erfordert die tatsächliche Anwesenheit des D-Arztes in der Praxis z. B. auch am Mittwochnachmittag. In Pausen ist eine Abwesenheit nur dann akzeptabel, wenn der D-Arzt z. B. bei Notfällen kurzfristig herbeigerufen wird.

\section{Probleme bei der praktischen Umsetzung}

Insbesondere durch das Vertragsarztrechtsänderungsgesetz werden Ärzten inzwischen weitgehende Freiheiten bei der Wahl des Niederlassungsortes oder auch der Eröffnung von Zweigpraxen eingeräumt. Auch bei einer Tätigkeit als Belegarzt an einem Krankenhaus oder beim ambulanten Operieren außerhalb der eigenen Praxis sind Konflikte mit dem Grundsatz der persönlichen Leistungserbringung bzw. der persönlichen Präsenzbereitschaft für den D-Arzt, nicht jedoch für den H-Arzt, denkbar. Für diese Fälle bietet $\$ 24$ Abs. 4 des Ärztevertrags die Lösung des „ständigen Vertreters“, der von den Landesverbänden auf Antrag anerkannt werden kann, wenn er über dieselbe fachliche Befähigung wie der D-Arzt selbst verfügt. Dies gilt sowohl für niedergelassene als auch für Ärzte im Krankenhaus. Gerade in der Niederlassung werden heute jedoch zunehmend Gemeinschaftspraxen gebildet. Erfüllen mehrere Ärzte die Anforderungen für die Beteiligung als D-Arzt, steht der gesonderten Zulassung jedes Einzelnen in aller Regel nichts entgegen. Sie können sich dann auch zu jeder Zeit gegenseitig vertreten, wobei natürlich gewährleistet sein muss, dass während der Präsenzzeiten in jeder Praxis bzw. Zweig-

Trauma Berufskrankh 2007 · 9[Suppl 3]: S346-S348 DOI 10.1007/s10039-007-1302-8

(c) Springer Medizin Verlag 2007

\section{H.-J. Wirthl \\ Die persönliche Leistungserbringung in Klinik und Praxis}

\section{Zusammenfassung}

Die Träger der gesetzlichen Unfallversicherung haben mit allen geeigneten Mitteln den durch einen Arbeitsunfall verursachten Gesundheitsschaden zu beseitigen oder zu verbessern, seine Verschlimmerung zu verhüten und seine Folgen zu mildern. Sie haben dazu alle Maßnahmen zu treffen, durch die eine möglichst frühzeitig einsetzende, sachgemäße und ggf. besondere unfallmedizinische Heilbehandlung gewährleistet wird. Zu diesem Zweck können sie die von Ärzten und Krankenhäusern zu erfüllenden Voraussetzungen und die von diesen zu übernehmenden Pflichten festlegen. Bei den von den UV-Trägern beteiligten Durchgangs- und $\mathrm{H}$ Ärzten handelt es sich um Fachärzte mit be-

\section{Personal provision of services in clinical and private medicine}

\author{
Abstract \\ The payers under the accident insurance pro- \\ vided as part of the social services are re- \\ quired to apply all appropriate means to cor- \\ rect or improve impaired health caused by \\ an occupational accident, to prevent wors- \\ ening and to mitigate any sequelae. To this \\ end, they must do all in their power to guar- \\ antee the earliest possible start of an appro- \\ priate, and if necessary a special emergency, \\ treatment. They are entitled to lay down the \\ conditions required of the doctors and hos- \\ pitals involved and the duties incumbent up- \\ on them. Doctors qualified to work as emer- \\ gency doctors of either grade are specialists \\ with specific training in accident and emer- \\ gency medicine. The personal provision of \\ services is therefore an exceptional feature of
}

sonderer unfallmedizinischer Qualifikation. Die persönliche Leistungserbringung ist deshalb eine herausragende Besonderheit des bg-lichen Heilverfahrens, die dazu beiträgt, dass die Behandlung stets auf hohem Qualitätsniveau erbracht werden kann. Eine Delegation der Tätigkeit kann deshalb nur auf gesondert zugelassene Vertreter mit derselben fachlichen Qualifikation vorgenommen werden.

\section{Schlüsselwörter}

Heilverfahren · Durchgangsarztverfahren . $\mathrm{H}$-Arztverfahren · Persönliche Leistungserbringung - Anforderungen der gesetzlichen UV-Träger · Unfallärztliche Bereitschaft the treatment approved by the employers' 1 iability insurance associations, which contributes to making it possible for the treatment always to be provided at a high level of quality. This work cannot therefore be delegated except to specially approved representatives with the same specialist qualifications.

\section{Keywords}

Treatment - Procedure for emergency treatment by fully qualified $A \& E$ doctor . Procedure for emergency treatment by A\&E doctor with lower qualification - Personal provision of services - Requirements of the payers of legally required accident insurance . A\&E emergency call out 
praxis ein Arzt anwesend ist, der über die Beteiligung als D-Arzt verfügt.

\section{Fazit}

Der hohe Standard der fachlichen Anforderungen an D- und H-Ärzte gewährleistet die Behandlungsqualität. Die persönliche Leistungserbringung ist deshalb kein überkommenes Relikt aus der Vergangenheit, sondern weiterhin unentbehrlich für Ärzte in Niederlassung und am Krankenhaus. Dabei sind die besonderen Anforderungen an die unfallärztliche Präsenzbereitschaft unabdingbare Voraussetzung für die Beteiligung als D-Arzt am berufsgenossenschaftlichen Heilverfahren.

\section{Korrespondenzadresse}

\section{Ltd. Verw.-Direktor H.-J. Wirthl}

Landesverband Hessen-Mittelrhein und Thüringen der gewerblichen Berufsgenossenschaften, Wilhelm-Theodor-Römheld-Straße 15,

55130 Mainz

service@mainz.lvbg.de

Interessenkonflikt. Der korrespondierende Autor gibt an, dass kein Interessenkonflikt besteht. 